\section{Static and dynamic light scattering of healthy and malaria-parasite invaded red blood cells}

\author{
YongKeun Park, ${ }^{\mathrm{a}, *}$ Monica Diez-Silva, ${ }^{\mathrm{b}}$ Dan Fu, ${ }^{\mathrm{a}}$ \\ Gabriel Popescu, ${ }^{\mathrm{c}}$ Wonshik Choi, ${ }^{\mathrm{d}}$ Ishan Barman, \\ Subra Suresh, ${ }^{\mathrm{b}, \mathrm{e}}$ and Michael S. Feld ${ }^{\mathrm{a}}$ \\ ${ }^{a}$ Massachusetts Institute of Technology, G. R. Harrison \\ Spectroscopy Laboratory, 77 Massachusetts \\ Avenue, Cambridge, Massachusetts 02139 \\ ${ }^{\mathrm{b}}$ Massachusetts Institute of Technology, Department of \\ Materials Science and Engineering, 77 Massachusetts \\ Avenue, Cambridge, Massachusetts 02139 \\ 'University of Illinois at Urbana-Champaign, Beckman \\ Institute for Advanced Science and Technology, Department \\ of Electrical and Computer Engineering, 405 North \\ Mathews Avenue, Urbana, Illinois 61801 \\ ${ }^{\mathrm{d}}$ Korea University, Department of Physics, Anam-dong \\ Sungbuk-gu, Seoul, 136-701 Korea \\ e Massachusetts Institute of Technology, School of \\ Engineering, 77 Massachusetts Avenue, Cambridge, \\ Massachusetts 02139
}

Abstract. We present the light scattering of individual Plasmodium falciparum-parasitized human red blood cells (Pf-RBCs), and demonstrate progressive alterations to the scattering signal arising from the development of malaria-inducing parasites. By selectively imaging the electric fields using quantitative phase microscopy and a Fourier transform light scattering technique, we calculate the light scattering maps of individual Pf-RBCs. We show that the onset and progression of pathological states of the Pf-RBCs can be clearly identified by the static scattering maps. Progressive changes to the biophysical properties of the Pf-RBC membrane are captured from dynamic light scattering. (c) 2010 Society of Photo-Optical Instrumentation Engineers. [DOI: 10.1117/1.3369966]

Keywords: red blood cell; plasmodium falciparum; light scattering; malaria; electric field.

Paper 09414LRR received Sep. 14, 2009; revised manuscript received Feb. 23, 2010; accepted for publication Feb. 26, 2010; published online Apr. 5, 2010.

Static and dynamic light scattering techniques have been widely used to study the structure and dynamics of scattering objects. Static (elastic) light scattering measures the angular distribution of the scattering intensity to infer structural information of scatterers, which is utilized to probe the structure of living cells or tissues. ${ }^{1}$ Dynamic (quasielastic) light scattering is the extension of elastic light scattering to study dynamic inhomogeneous systems. ${ }^{2}$ Several techniques have been used to study light scattering, including photon correlation spectroscopy, ${ }^{17}$ diffuse wave spectroscopy, ${ }^{3}$ dynamic scattering microscopy, ${ }^{4}$ and single-cell partial-wave spectroscopy. ${ }^{5}$ Recently, Fourier transform light scattering (FTLS), which numerically calculates far-field scattering patterns from the

*Address all correspondence to: YongKeun Park, Massachusetts Institute of Technology, G. R. Harrison Spectroscopy Laboratory, 77 Massachusetts Avenue, Cambridge, Massachusetts 02139. E-mail: ykpark@mit.edu electric field (E-field) measurement, has been developed. ${ }^{6,7}$

Light scattering of red blood cells (RBCs) has also been extensively studied. Static light scattering of RBCs has been widely used in flow cytometry for measuring size and hemoglobin $(\mathrm{Hb})$ concentration distribution. ${ }^{8}$ Numerical models have been introduced to simulate static light scattering of RBCs. ${ }^{9}$ Dynamic light scattering has been used to study the dynamic motion of RBC membranes. ${ }^{10}$ The RBC membrane cortex is sufficiently soft and elastic, and it exhibits dynamic membrane fluctuations driven by both thermal and metabolic energies. ${ }^{11}$ These membrane fluctuations are influenced by mechanical properties of the membrane, and serve as an important indicator of RBC deformability, which in turn plays an important role in the evolution of a number of human diseases.

When invaded by the malaria-inducing parasite $P$. falciparum host RBCs undergo substantial changes. ${ }^{12,13}$ The parasite causes modifications in the structure, mechanical properties, and biochemical characteristics of the host RBCs. In response to these changes, the characteristic biconcave shape of the RBC is lost, the dynamics of membrane fluctuations are altered, and the $\mathrm{Hb}$ concentration in RBC cytoplasm decreases. These changes could affect both static and dynamic light scattering of Pf-RBCs. In this study, the E-field maps of individual Pf-RBCs were measured and utilized to determined both static and dynamic light scattering distributions. We show that the static light scattering patterns of Pf-RBCs are significantly different among different disease states at specific scattering angle windows. We also present dynamic light scattering to characterize transitions from intact membranes of healthy RBCs to modified membranes of Pf-RBCs.

We prepared RBCs in four different groups following the standard protocol ${ }^{14}$ : healthy RBCs, and three parasite maturation stages of Pf-RBCs with 14 to $24 \mathrm{~h}$ (late ring stage), 24 to $36 \mathrm{~h}$ (trophozoite stage), and 36 to $48 \mathrm{~h}$ (schizont stage) after the invasion of the $P$. falciparum merozoite. We first measured the light-intensity scattering patterns of individual Pf-RBCs by FTLS. ${ }^{6,7}$ The physical principle of FTLS is to measure the complex E-field at the image plane and then numerically propagate this E-field to the far-field scattering plane. To quantitatively measure the E-field maps of PfRBCs, we employed diffraction phase microscopy (DPM). ${ }^{15,16}$ For each individual RBC, we measured the E-field $E(x, y ; t)$ $=A(x, y ; t) \exp [j \phi(x, y ; t)]$, where $A(x, y ; t)$ is the amplitude, and $\phi(x, y ; t)$ is the phase at a position of $(x, y)$ at time $t$ [Figs. 1(a) and 1(b)]. This E-field is divided by the E-field without samples to normalize the intensity of the incident beam. From the measured E-field, the far-field scattering intensity is calculated by using a 2-D Fourier transform,

$$
\begin{aligned}
I\left(q_{x}, q_{y} ; t\right)= & \mid \frac{1}{2 \pi} \iint E(x, y ; t) \exp \left(-j q_{x} x\right) \\
& \times\left.\exp \left(-j q_{y} y\right) d x d y\right|^{2}
\end{aligned}
$$

where $q_{x}, q_{y}$ are spatial frequencies. The time-averaged (static) scattering intensity map $\bar{I}\left(q_{x}, q_{y}\right)=\left\langle I\left(q_{x}, q_{y} ; t\right)\right\rangle$ of a healthy RBC is shown in Fig. 1(c).

1083-3668/2010/15(2)/020506/3/\$25.00 @ 2010 SPIE 

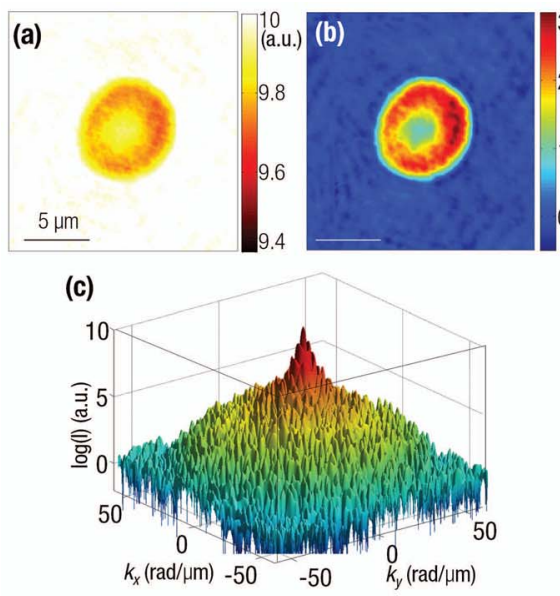
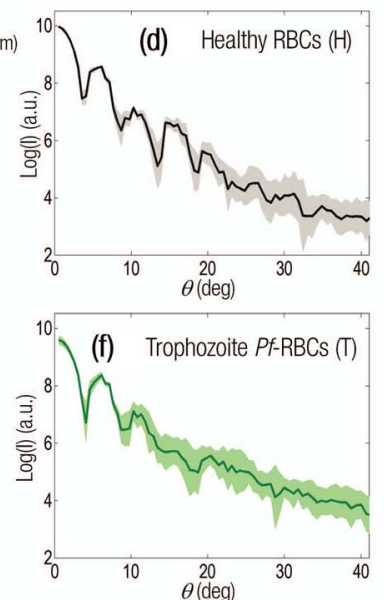
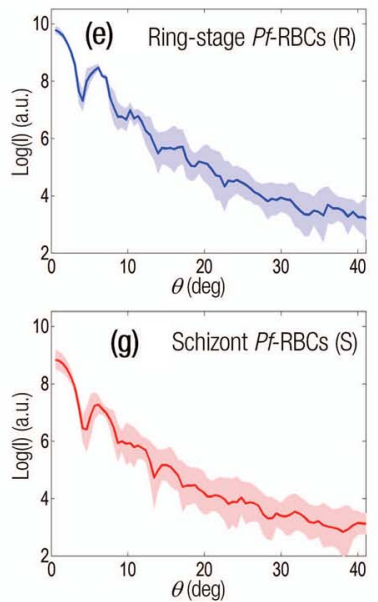

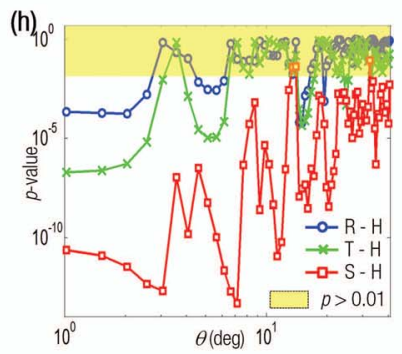

Fig. 1 (a) Amplitude and (b) phase map of a healthy RBC. (c) The retrieved light scattering pattern of the same cell. Light-intensity scattering patterns of (d) healthy RBCs, (e) ring, (f) trophozoite, and (g) schizont stage of Pf-RBCs. (h) p-values of scattering patterns (different intraerythrocytic stages of Pf-RBCs are compared to the healthy RBCs).

We then retrieved the scattering patterns of Pf-RBCs at different pathological states. By utilizing the FTLS method, we can easily locate an individual Pf-RBC in the field of view and measure both imaging and scattering maps of the sample simultaneously. To our knowledge there has been no prior study of light scatterings of Pf-RBCs. The light scattering patterns with respect to the different parasitization stages are plotted as a function of scattering angle from 0 to $41 \mathrm{deg}$ after azimuthal average and converting the spatial frequency to angle, by using the relation $q=2 \pi n \sin \theta / \lambda$, where $\lambda$ is the wavelength of the laser, and $n$ is the refractive index of the medium [Figs. 1(d) $-1(\mathrm{~g})]$. The scattering patterns of healthy RBCs show the distinct periodic oscillatory features, which are consistent with previous measurements and simulations. ${ }^{8,9}$ For the Pf-RBCs, not only did the oscillatory patterns change, especially for higher angles ( $>10 \mathrm{deg}$ ), but the scattering intensities at $0 \mathrm{deg}$ also decreased by $16.6 \%$ (ring-), $30.7 \%$ (trophozoite-), and $65.7 \%$ (schizont stage) compared to healthy RBCs. This indicates clearly identifiable decreases in refractive index between the RBC cytoplasm and the medium, which is consistent with the previous $\mathrm{Hb}$ concentration measurements. ${ }^{14}$ This trend can be explained by the reduction in $\mathrm{Hb}$ concentration in the cytoplasm as the invading parasite digests $\mathrm{Hb}$ from its host $\mathrm{RBCs}$. To test whether these scattering patterns can distinguish the different parasite maturation stages from healthy RBCs, we performed the two-tailed Mann-Whitney rank sum tests [Fig. 1(h)]. p-values of PfRBCs versus healthy RBCs were smaller than 0.01 for the low scattering angle range (0 to $2 \mathrm{deg}$ and 4 to $5 \mathrm{deg}$ ). At this angle range, each parasite development stage of Pf-RBCs can be statistically differentiated based on the static light scattering signals; averaged $p$-values are 0.005 (ring versus trophozoite stage) and $<10^{-7}$ (trophozoite versus schizont stage). This suggests that the presence of the Pf-RBCs, and even the particular parasite development stage, can be clearly detected by static light scattering techniques at these specific angles.

To measure the dynamic light scattering in individual PfRBCs, we measured the E-field maps of Pf-RBCs for about $2 \mathrm{~s}$ at 120 frames per s. For each E-field map, we calculated the scattering intensity patterns as described before, which provide the dynamic light scattering information. To quantitatively analyze the dynamic scattering signals, we calculated normalized temporal autocorrelation functions of the intensity fluctuations $\langle I(\tau) I(0)\rangle /\langle I(\tau)\rangle^{2}[$ Fig. 2(a)]. The spectrum of the
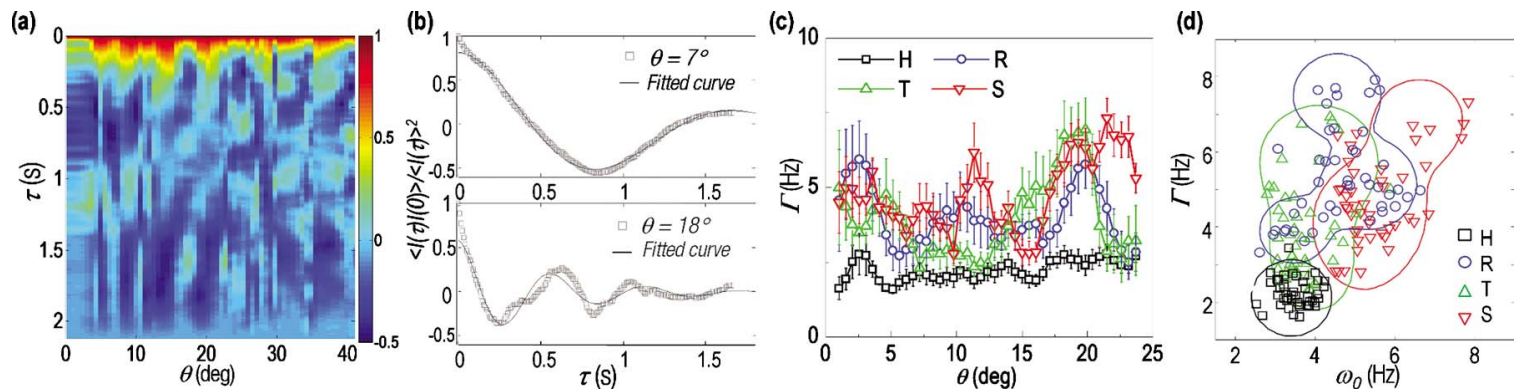

Fig. 2 Dynamic light scattering of Pf-RBCs. (a) Normalized temporal autocorrelations of a healthy RBC as a function of decay time and scattering angle. (b) Normalized temporal autocorrelation of a healthy RBC at two representative scattering angles and fitted curves. (c) Line width $\Gamma$ extracted from healthy and Pf RBCs as a function of scattering angle. Symbols represent the mean value and the error bars indicate the standard error for 15 RBCs. (d) Scatter plot of $\omega_{0}$ versus $\Gamma$ taken for healthy and Pf RBCs. Each symbol represents mean values of 15 RBCs at scattering angles (0 to $21 \mathrm{deg}$ ). Lines indicate the boundaries of each group. 
scattered light can be described approximately as Lorentzian, having a peak frequency $\omega_{0}$ and a line width $\Gamma$, and its autocorrelation is described as a damped cosine function ${ }^{17}$ :

$$
G(\tau)=A+B \cos \left(\omega_{0} \tau+\varphi\right) \exp (-\Gamma \tau) \exp \left(-\beta^{2} \tau^{2}\right)
$$

The phase term $\varphi$ is added to include the deviation of the spectrum from an exact Lorentzian form, and the $\beta$ term represents the instrumental variations. For individual RBCs, the intensity autocorrelation was calculated at each scattering angle, from which $\omega_{0}$ and $\Gamma$ were retrieved by fitting to Eq. (1) [Fig. 2(b)]. The extracted values of $\Gamma$ showed significant differences between healthy RBCs and Pf-RBCs for the scattering angles 0 to $25 \mathrm{deg}$. Peak frequencies $\omega_{0}$ did not show significant differences between the pathological states (results not shown). The $\omega_{0}$ and $\Gamma$ values for the scattering angles 0 to $21 \mathrm{deg}$ are plotted in Fig. 2(d). Compared to Pf-RBCs, healthy RBCs show small values for $\omega_{0}$ and $\Gamma$ (around 2 and $3.5 \mathrm{~Hz}$, respectively), and small cell-to-cell variations. However, the values of Pf-RBCs span larger areas (2 to $8 \mathrm{~Hz}$ for $\omega_{0}$ and 1 to $8 \mathrm{~Hz}$ for $\Gamma$ ). We employed the support vector machines method, a machine learning technique for classification, ${ }^{18}$ to generate contour lines that separate different stages of Pf-RBCs [Fig. 2(d)]. To better address the alterations in the dynamic light scattering signals accompanied with the progress of intraerythrocytic stages of $P$. falciparum, we performed a t-test using a new complex parameter $S$ $=\omega_{0}+i \Gamma$. The $p$-values are $<10^{-17},<10^{-8}$, and $<10^{-5}$ for ring versus Healthy, trophozoite versus ring, and schizont versus trophozoite, respectively. For a simple flat lipid bilayer model, $\omega_{0}$ is directly related to membrane tension and $\Gamma$ to viscosity of medium. ${ }^{17}$ However, for a complex curved RBC membrane cortex, there is no theoretical model to relate dynamic light scattering signals, and thus it is not straightforward to quantitatively extract mechanical parameters from the values for $\omega_{0}$ and $\Gamma$. Nevertheless, we can qualitatively interpret that the increase of $\omega_{0}$ can result from increased stiffness or decreased mass for membrane cortex, and the increase in $\Gamma$ from increased viscosity. Taken together, this result indicates that mechanical properties of RBC membrane undergo significant modifications during pathological states induced by parasite maturation within the RBC, which can result from the stiffening of the membrane cortex and the formation of membrane-bound proteins.

In conclusion, by analyzing the E-field images with the FTLS technique, we report static and dynamic light scattering maps of individual Pf-RBCs, and demonstrate a novel methodology to identify and distinguish pathological intraerythrocytic stages of $P$. falciparum malaria. The static scattering maps of individual cells showed that the scattering pattern can distinguish the specific disease states. The dynamic scattering results suggest alterations in mechanical properties of Pf-RBC membranes, consistent with other independent experimental measurements that employed optical tweezers. ${ }^{12}$ These results demonstrate that light scattering cannot only detect Pf-RBCs, but can also be utilized to assess biological questions about the pathophysiology of the malaria infection, as well as other RBC-related diseases.

\section{Acknowledgments}

This research was supported by the National Institutes of Health (P41-RR02594-18). Park was supported by a Samsung Scholarship. Diez-Silva and Suresh acknowledge support from the Interdisciplinary Research Group on Infectious Diseases, which is supported by the Singapore-MIT Alliance for Research and Technology and by the National Institutes of Health (1R01HL094270-01A1).

\section{References}

1. R. Gurjar, V. Backman, L. Perelman, I. Georgakoudi, K. Badizadegan, I. Itzkan, R. Dasari, and M. Feld, "Imaging human epithelial properties with polarized light-scattering spectroscopy," Nat. Med. 7(11), 1245-1248 (2001)

2. B. Berne and R. Pecora, Dynamic Light Scattering: with Applications to Chemistry, Biology, and Physics, Dover Publications, Mineola, New York (2000).

3. D. Pine, D. Weitz, P. Chaikin, and E. Herbolzheimer, "Diffusing wave spectroscopy," Phys. Rev. Lett. 60(12), 1134-1137 (1988).

4. M. Amin, Y. Park, N. Lue, R. Dasari, K. Badizadegan, M. Feld, and G. Popescu, "Microrheology of red blood cell membranes using dynamic scattering microscopy," Opt. Express 15(25), 17001-17009 (2007).

5. H. Subramanian, P. Pradhan, Y. Liu, I. Capoglu, X. Li, J. Rogers, A Heifetz, D. Kunte, H. Roy, A. Taflove, and V. Backman, "Optical methodology for detecting histologically unapparent nanoscale consequences of genetic alterations in biological cells," Proc. Natl. Acad. Sci. U.S.A. 105(51), 20118 (2008).

6. W. Choi, C. Yu, C. Fang-Yen, K. Badizadegan, R. Dasari, and M Feld, "Field-based angle-resolved light-scattering study of single live cells," Opt. Lett. 33(14), 1596-1598 (2008).

7. H. Ding, Z. Wang, F. Nguyen, S. Boppart, and G. Popescu, "Fourier transform light scattering of inhomogeneous and dynamic structures," Phys. Rev. Lett. 101(23), 238102 (2008).

8. D. Tycko, M. Metz, E. Epstein, and A. Grinbaum, "Flow-cytometric light scattering measurement of red blood cell volume and hemoglobin concentration," Appl. Opt. 24(9), 1355-1365 (1985).

9. J. Steinke and A. Shepherd, "Comparison of Mie theory and the light scattering of red blood cells," Appl. Opt. 27(19), 4027-4033 (1988).

10. R. Tishler and F. Carlson, "Quasi-elastic light scattering studies of membrane motion in single red blood cells," Biophys. J. 51(6), 993 997 (1987).

11. Y. Park, C. Best, T. Auth, N. Gov, S. Safran, G. Popescu, S. Suresh, and M. Feld, "Metabolic remodeling of the human red blood cell membrane," Proc. Natl. Acad. Sci. U.S.A. 107(4), 1289 (2010).

12. S. Suresh, J. Spatz, J. P. Mills, A. Micoulet, M. Dao, C. T. Lim, M. Beil, and T. Seufferlein, "Connections between single-cell biomechanics and human disease states: gastrointestinal cancer and malaria," Acta Biomater. 1(1), 15-30 (2005).

13. J. P. Mills, M. Diez-Silva, D. J. Quinn, M. Dao, M. J. Lang, K. S. W. Tan, C. T. Lim, G. Milon, P. H. David, O. Mercereau-Puijalon, S Bonnefoy, and S. Suresh, "Effect of plasmodial RESA protein on deformability of human red blood cells harboring Plasmodium falciparum," Proc. Natl. Acad. Sci. U.S.A. 104(22), 9213-9217 (2007).

14. Y. K. Park, M. Diez-Silva, G. Popescu, G. Lykotrafitis, W. Choi, M. S. Feld, and S. Suresh, "Refractive index maps and membrane dynamics of human red blood cells parasitized by Plasmodium falciparum," Proc. Natl. Acad. Sci. U.S.A. 105(37), 13730 (2008).

15. G. Popescu, T. Ikeda, R. R. Dasari, and M. S. Feld, "Diffraction phase microscopy for quantifying cell structure and dynamics," $O p t$ Lett. 31(6), 775-777 (2006).

16. Y. K. Park, G. Popescu, K. Badizadegan, R. R. Dasari, and M. S. Feld, "Diffraction phase and fluorescence microscopy," Opt. Express 14(18), 8263-8268 (2006).

17. D. Byrne and J. Earnshaw, "Photon correlation spectroscopy of liquid interfaces. I. Liquid-air interfaces," J. Phys. D 12(7), 1133-1144 (1979).

18. C. Bishop and M. Tipping, "Variational relevance vector machines," in Proc. 16th Conf. on Uncertainty in Artificial Intelligence, pp. 4653, Morgan Kaufmann, San Francisco (2000). 